\title{
Role of Local Festivals on Network Formation among a Variety of Residents in a Community
}

$\operatorname{AUTHOR}(\mathrm{S})$ :

Kotani, Hitomu; Yokomatsu, Muneta

\section{CITATION:}

Kotani, Hitomu ... [et al]. Role of Local Festivals on Network Formation among a Variety of Residents in a Community. 2015 IEEE International Conference on Systems, Man, and Cybernetics, SMC 2015 2015: 832-839

ISSUE DATE:

2015-10

URL:

http://hdl.handle.net/2433/217788

\section{RIGHT:}

(C) 2015 IEEE. Personal use of this material is permitted. Permission from IEEE must be obtained for all other uses, in any current or future media, including reprinting/republishing this material for advertising or promotional purposes, creating new collective works, for resale or redistribution to servers or lists, or reuse of any copyrighted component of this work in other works.; この論文は出版社版でありません。引用の際には出版社版をご確認ご利用ください。; This is not the published version. Please cite only the published version. 


\section{Role of Local Festivals on Network Formation among a Variety of Residents in a Community}

\author{
Hitomu Kotani \\ Department of Urban Management \\ Kyoto University \\ Kyoto, Japan \\ hitomukotani@drs.dpri.kyoto-u.ac.jp
}

\author{
Muneta Yokomatsu \\ Disaster Prevention Research Institute \\ Kyoto University \\ Kyoto, Japan \\ yoko@drs.dpri.kyoto-u.ac.jp
}

\begin{abstract}
Community activities, such as local festivals, which get residents to collaborate with each other, can enable them to start interacting with others whom they have never interacted with before. This is because festivals exist in a context that is independent of daily jobs or hobbies of residents. This study develops a social network model with the assumption that (i) each player's knowledge and experience is endogenously determined through a network and (ii) players have various preferences for the heterogeneity of this knowledge and experience. We carry out numerical simulations to illustrate the dynamic process of interaction among players. Furthermore, we investigate the dynamic effect of exogenous link addition by festivals on network formation and utility of each player, and evaluate the value of festivals.

Index Terms-Social network, festival, endogenous knowledge and experience, heterogeneous preference
\end{abstract}

\section{INTRODUCTION}

Through the application of the social network model, this study analyzes the dynamic process of the interaction between residents in a community where local "festivals" exist. Social network models have been used to analyze the interaction between various individuals and collective behavior. The models are useful in analyzing "network externality", where a tie between two individuals affects utility of other individuals who are either directly or indirectly connected with one of the two. For instance, let us consider the case where individuals $\mathrm{A}$ and $\mathrm{B}$ are connected. If individual $\mathrm{B}$ starts to interact with individual $\mathrm{C}$, then individual $\mathrm{A}$ is more likely to make a tie with individual $\mathrm{C}$. This is because the common friend $\mathrm{B}$ has the potential of introducing $\mathrm{A}$ to $\mathrm{C}$. Using this kind of "network externality" as a key concept, much of the research has discussed many kinds of phenomena such as the information flow and the coordinated actions under the framework of network models thus far (e.g. [1] - [4]).

This study analyzes the dynamics of the network externality generated by local "festivals". In the domain of sociological studies, it has been revealed that unlike day-to-day life, special occasions influence individual's psychological process and social integration (e.g. [5] - [7]). Therefore, in dealing with connections among residents in a community, local "festivals" should be taken into consideration as representation of exceptional times. In general, festivals provide an opportunity for people to meet and cooperate with others with distinct occupations, ages, nationalities, and so on. Through these opportunities, even in day-to-day life, some people start to interact with others who would they have never met otherwise. In other words, this study assumes that festivals provide people with the opportunity to make unintentional connections even in ordinary situations.

In light of the role of festivals, how do festivals affect network structure and utility derived from such networks? What is the value of festivals in terms of social network formation? To answer these critical research questions, we develop a model on network formation in a community with festivals, and then, through numerical simulations, we analyze the dynamics of the network characteristics and utility.

Much of the previous literature on network formation is mainly under three assumptions: 1) exogenous types or characteristics, 2) homophily, and 3) the network formation based on the notion of improving paths.

For example, Johnson and Gilles [8], Droste et al. [9], and Iijima and Kamada [10] deal with network formation based on homophily, the socio-psychological tendency of people to interact with others similar to oneself (e.g. [11] - [12]). In these network studies, it is assumed that types or characteristics, which are given exogenously to each player, determine (social) distance between players. A pair of the players with a smaller social distance pays less cost or obtains more benefit of maintaining the link. Under these assumptions, the process of integration of a community, for example, how and why people with similar race tend to live close, has been discussed.

In addition, many social network models consider the improving path as the basis for the network formation based on the rational player [13]. In these models, player $i$ and $j$ add the link $i j$ if the two players involved both agree to its addition, with at least one of the two strictly benefiting from the addition of the link. Meanwhile, the two delete the link if at least one of two players involved in the link strictly benefits from its deletion. So, the formation of a link requires the consent of both players, but severance can be done unilaterally. This individual optimization process leads to networks called pairwise stable networks. 
On the contrary, our model revises the above three assumptions. We consider that 1) players have the knowledge and experience that are determined endogenously through network and moreover these endogenous variables determine the "homogeneity" and "heterogeneity" between players. We also note that 2) each player has a preference not only for the homogeneity but also for the heterogeneity of the endogenous knowledge and experience and that 3 ) festivals create opportunities to add a link independently of player's evaluation based on utility function in their day-to-day life.

1) This study focuses on endogenously determined knowledge and experience of players rather than exogenously determined types or characteristics. This is because we suppose that whether or not to maintain connections would essentially depend on not just exogenous types or characteristics of other players, but their endogenously determined knowledge and experience. We also assume that this knowledge and experience can be created through their interaction with others. In fact, people can learn and obtain new knowledge and experience from others who they meet. Under this assumption, both "homogeneity" and "heterogeneity" of knowledge and experience between players are also determined endogenously through the network.

2) This study considers "heterophily", while much literature on network formation focuses on "homophily". Essentially, from a viewpoint of exchange of knowledge and experience, some prefer to associate with similar people; others prefer to associate with diverse others. We focus on a community where players who prefer homogeneity and players who prefer heterogeneity coexist; that is, two types of preferences for knowledge and experience coexist. Then we analyze how players with various preferences create networks. Needless to say, there are several papers that focus on heterophily (e.g. Currarini et al. [14] and $\mathrm{Fu}$ et al. [15]). However, these papers do no more than assume the existence of just heterophilous players or focus on preference for heterogeneity of exogenous types or characteristics.

Finally, 3) this study focuses on the exceptional times produced by festivals. As previously mentioned, festivals provide the opportunity for people to interact with others who they would have never met otherwise. Our model assumes that festivals create links that would not necessarily be created under utility function in day-to-day life. In other words, we interpret festivals as events that add a link without mutual consent under utility function in day-to-day life, and then add this link formation that festivals generate as exogenous shock to the dynamics based on the improving path. We analyze how such festivals affect the dynamic process of one-to-one link formation in a community. Undoubtedly, many aspects of festivals have been studied in the field of social network theory. However, we do not know of any studies that model the link formation in exceptional times of festivals as mentioned above and analyze its dynamic effect on formation of social networks. In contrast, this study constructs the social network model with a focus on the three aforementioned features.

The remainder of the paper is organized as follows. In
Section 2 we present the model of stochastic dynamics of network formation. In Section 3 we analyze network formation without festivals by means of numerical simulations. In Section 4 we extend the model to include festivals and analyze the dynamic effect of link addition by festivals. Finally, Section 5 concludes and describes future work.

\section{Social Network Formation CONSIDERING Preferences For Homogeneity ANd Heterogeneity OF KNOWLEDGE AND EXPERIENCE}

\section{A. The Model}

Let $N=\{1, \cdots, n\}$ be the finite set of players $(n>2)$. The network relations among the players are represented by graphs whose nodes represent the players and whose links capture the connections between the players. A network $g$ is a set of links between players in $N$. ij represents the link connecting players $i$ and $j$. If $i j \in g$, then players $i$ and $j$ are directly connected in the network $g$, while if $i j \notin g$, then players are not directly connected. For instance, if $n=3$ and there are directed links between players 1 and 2 and players 2 and 3 , the network is written as $g=\{12,23\}$. Player $i$ 's neighbors are $j \in N$ with $i j \in g$. Formally, the set of $i$ 's neighbors in $g$, denoted by $N_{i}(g)$, is defined as $N_{i}(g)=\{j \in N \mid i j \in g\}$. Player $i$ 's degree is the number of $i$ 's neighbors, which is defined as $d_{i}(g)=\left|N_{i}(g)\right|$.

Each player is assumed to have knowledge and experience that are endogenously determined through her/his neighbors. Thus, how similar, in other words, how "homogeneous" player $j$ 's knowledge and experience are for player $i$ would heavily depend on how many neighbors the players share. Meanwhile, how different, in other words, how "heterogeneous" player $j$ 's knowledge and experience are for player $i$ would heavily depend on how many $j$ 's neighbors are not $i$ 's neighbors. We assume that the more (less) neighbors player $i$ shares with player $j$, the more homogeneous (heterogeneous) the pair is. This assumption seems to be natural because it is more likely that if $i$ shares more (less) neighbors with $j$, then $i$ gains similar (different) knowledge and experience with (from) $j$ through her/his neighbors.

Hence, in terms of knowledge and experience, we call the part in which players $i$ and $j$ resemble as "homogeneity $S_{i j}$ ", while we call the part in which players $i$ and $j$ vary as "heterogeneity $D_{i j}$ ", which we represent respectively as follows:

$$
\begin{gathered}
S_{i j}=\left|N_{i}(g) \cap N_{j}(g)\right|, \\
D_{i j}=\left|N_{j}(g) \backslash\left(N_{i}(g) \cup\{i\}\right)\right| .
\end{gathered}
$$

$S_{i j}$ is defined as the number of elements of the intersection of the sets of neighbors of $i$ and $j$, while $D_{i j}$ is defined as the number of elements of the complement of the set of $i$ 's neighbors relative to the set of $j$ 's neighbors.

We will consider preferences for homogeneity and heterogeneity. Let $x_{i} \in\{0,1\}$ be a parameter that represents each player's preference for heterogeneity. If a player has $x_{i}=0$, then the player has a preference for homogeneity $S_{i j}$. If a 
player has $x_{i}=1$, then the player has a preference for heterogeneity $D_{i j}$. In what follows, we call the former players as "homophilous" and the latter players as "heterophilous".

Assuming that each player benefits from her/his neighbors' homogeneity and heterogeneity and the number of her/his neighbors, we now define the utility function as follows:

$$
u_{i}(g)=\left(\sum_{j \in N_{i}(g)} b\left(x_{i}, D_{i j}, S_{i j}\right)\right)-c\left(d_{i}\right) \cdot d_{i},
$$

where $b(\cdot)$ in the first term represents the benefit that $i$ obtains from a link with $j$, and $c\left(d_{i}\right)$ in the second term represents the cost for $i$ of maintaining a link. In general, the larger the number of $i$ 's neighbors is, the more the schedule adjustment and the opportunity cost seem to increase for $i$, so $c\left(d_{i}\right)$ is assumed to be non-decreasing function, that is, $\frac{d c\left(d_{i}\right)}{d d_{i}} \geq 0$.

\section{B. The Dynamic Process}

$n$ players play a game repeatedly. Let $g^{t}$ denote the network at the beginning of period $t(\geq 1)$. In an arbitrary period $t$, given $g^{t}$, players decide to add or delete links.

Our dynamic process of link formation belongs to the stochastic dynamic process, which has been widely used in many papers (e.g., Jackson and Watts [13], [16]). The stochastic dynamic process is based on the notion of improving path accompanied with exogenous randomness. This means that the process is subject to evolutionary pressure, namely, bounded rationality: the inertia, myopic and error/mutation hypothesis. In the situation we consider i) not all players need to react instantaneously to their environment (the inertia hypothesis), ii) when players react, they react myopically (the myopic hypothesis), and iii) there is a small probability that players change their strategies at random (the error/mutation hypothesis). For example, without any error/mutation, one of equilibrium is locked in at random by externality, whereas under the stochastic process, the network continues to change indefinitely and to visit each network over time, so in the long run the process identifies the most robust or easy-to-reach networks.

The dynamic process in period $t$ consists of the following 4 steps, proposed by Jackson and Watts [13].

$1 \mathrm{st}$

A pair of players $i j$ is randomly identified with fixed 2nd probability distribution $\left\{p_{i j}\right\}\left(p_{i j}>0\right)$.

The rest of the players (i.e. players except the pair $i j$ ) does not react instantaneously to their environment, in other words, does not adjust their links (the inertia hypothesis).

$3 r d$

The pair $i j$, identified in the 1st step, myopically decides whether to add or delete the link $i j$, following the rule which is shown below (the myopia hypothesis). They make this choice based on the assumption that players (including themselves) will have the same links (except the link $i j$ ) as at the beginning of this period.

3-1 In case that the link $i j$ is not in the network $g^{t}$, it is added if at least one player's utility increases and the other player's does not decrease. Otherwise, it is not added.

3-2 In case that the link $i j$ is already in the network $g^{t}$, it is deleted if either player would benefit from its removal. Otherwise, it is kept being connected.

4 th

After the choice is made, there is some small probability $\epsilon(0<\epsilon<1)$ that an error/mutation occurs (the error/mutation hypothesis). Hence, the choice is not reversed with probability $1-\epsilon$ and is reversed with probability $\epsilon$. This process determines the network $g_{1}^{t}$. To be more specific, the network $g_{1}^{t}$ is obtained as follows.

- In case $i j \notin g^{t}$

If $u_{i}\left(g^{t}+i j\right) \geq u_{i}\left(g^{t}\right)$ and $u_{j}\left(g^{t}+i j\right) \geq u_{j}\left(g^{t}\right)$ with one inequality strict, then $g_{1}^{t}=g^{t}+i j$ with probability $(1-\epsilon)$ and $g^{t}$ with $\epsilon$. Otherwise, $g_{1}^{t}=$ $g^{t}$ with probability $(1-\epsilon)$ and $g^{t}+i j$ with $\epsilon$.

- In case $i j \in g^{t}$ If $u_{i}\left(g^{t}-i j\right)>u_{i}\left(g^{t}\right)$ and/or $u_{j}\left(g^{t}-i j\right)>$ $u_{j}\left(g^{t}\right)$, then $g_{1}^{t}=g^{t}-i j$ with probability $(1-\epsilon)$ and $g^{t}$ with probability $\epsilon$. Otherwise, $g_{1}^{t}=g^{t}$ with probability $(1-\epsilon)$ and $g^{t}-i j$ with probability $\epsilon$.

The network $g_{1}^{t}$ that is determined through the above process is the network at the end of period $t$. This network is also the network at the beginning of period $t+1$. After period $t+1$, the above process is repeated.

Note that in the 3rd step you can see the asymmetry of adding and deleting a link. This represents that the formation of a link requires the consent of both players, but severance can be done unilaterally.

\section{NumeriCAl Simulation}

\section{A. Functions and Parameters}

In this section we consider the case without festivals as a basic model. Then, we examine the dynamics of each homophilous and heterophilous player's degree, the number of homophilous and heterophilous players in the degree, and her/his net utility obtained from the links by means of numerical simulation.

We specify the functions and parameters as follows:

$$
\begin{aligned}
& b\left(x_{i}, S_{i j}, D_{i j}\right)=x_{i} D_{i j}+\left(1-x_{i}\right) S_{i j}, \\
& c\left(d_{i}\right)=\bar{c}, \\
& p_{i j}=\frac{1}{\left(\begin{array}{c}
n \\
2
\end{array}\right)}, g^{1}=\emptyset, \\
& n=20, t=3000, \bar{c}=0.1, \\
& \epsilon=0.025 .
\end{aligned}
$$




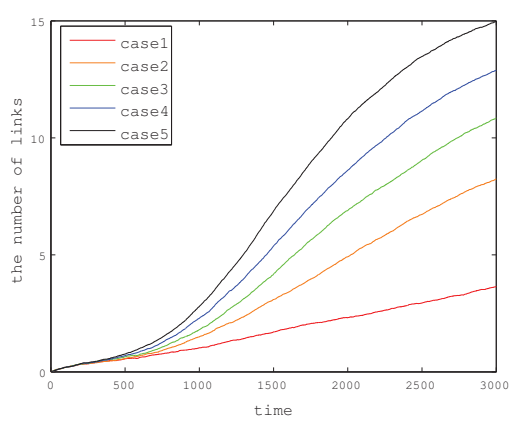

Fig. 1. One homophilous player's degree

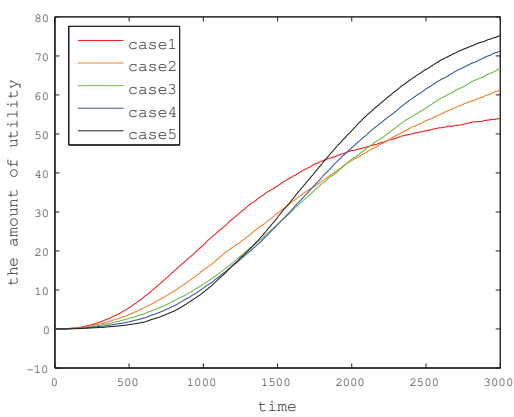

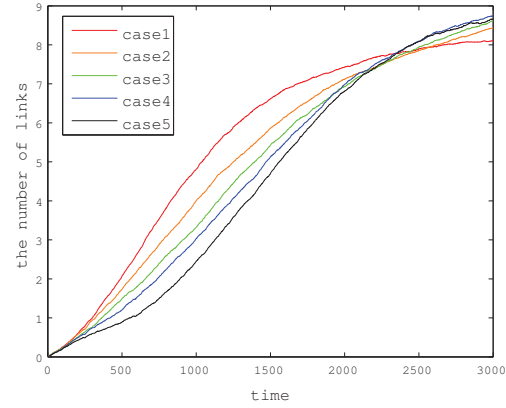

Fig. 2. One heterophilous player's degree

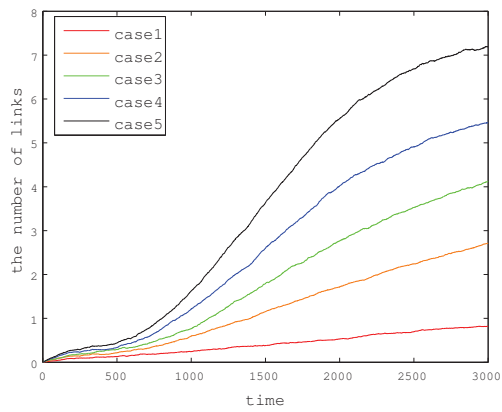

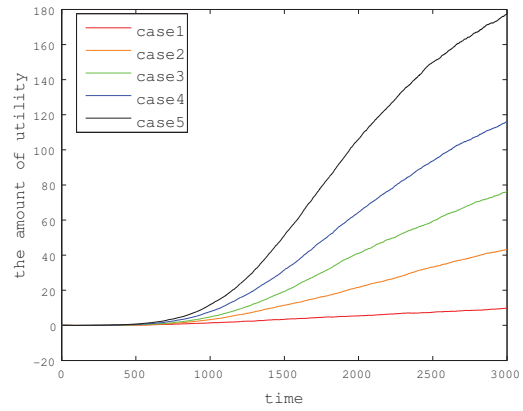

Fig. 3. One homophilous player's net utility obtained from her/his links

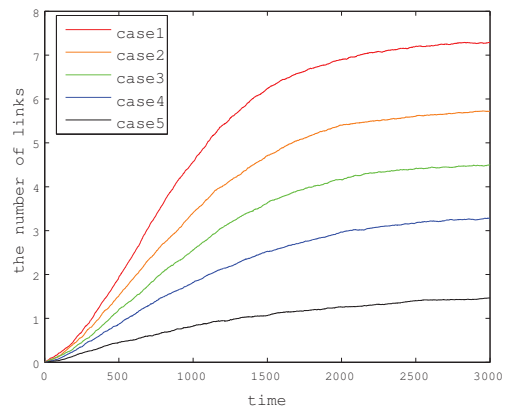

Fig. 4. One heterophilous player's net utility ob- Fig. 5. The number of links that one heterophilous Fig. $6 . \quad$ The number of links that one heterophilous tained from her/his links player has with homophilous players player has with heterophilous players

The benefit $b\left(x_{i}, S_{i j}, D_{i j}\right)$, which $i$ obtains from a link with $j$, is a linear function where homogeneity $S_{i j}$ is weighted with $1-x_{i}$ and heterogeneity $D_{i j}$ is weighted with $x_{i}$.

The cost $c\left(d_{i}\right)$, which represents the cost for a player of maintaining a link, remains constant regardless of the increase of the number of $i$ 's neighbors. Under $\bar{c}=0.1$, homophilous player $i$ benefits from adding a link with player $j$ sharing at least one same neighbor, while heterophilous player $i$ benefits from adding a link with player $j$ having at least one neighbor who is not $i$ 's neighbor.

The fixed probability $p_{i j}$ for the 1 st step in the dynamic process is uniformly distributed. The initial network $g^{1}$ is the empty network.

In the analysis, let us examine the following 5 cases, whose proportion of homophilous and heterophilous players is different. The five different cases would let us deeply understand how various players interact with each other.

case 1

$25 \%(i=1, \cdots, 5)$ homophilous players, and $75 \%(i=6, \cdots, 20)$ heterophilous players.

case 2

$40 \%(i=1, \cdots, 8)$ homophilous players, and $60 \%(i=9, \cdots, 20)$ heterophilous players. case 3

$50 \%(i=1, \cdots, 10)$ homophilous players, and $50 \%(i=10, \cdots, 20)$ heterophilous players. case 4

$60 \%(i=1, \cdots, 12)$ homophilous players, and $40 \%(i=13, \cdots, 20)$ heterophilous players.

case 5

$75 \%(i=1, \cdots, 15)$ homophilous players, and $25 \%(i=16, \cdots, 20)$ heterophilous players.

We run a Monte Carlo simulation with 1,000 iterations for each case, and examine its mean in the analysis.

\section{B. Results}

Simulations result show that each homophilous and heterophilous player's degree and their utility increase as time goes on (Fig. 1-4).

Let us consider cases 1-5, dividing the time into "an early stage", where links are connected mainly by error/mutation, and "a later stage", where links are connected on the basis of an early stage. In what follows, for simplifying the notation, we express a link between homophilous players as "homo-homo", a link between heterophilous players as "hetero-hetero", and a link between a homophilous player and a heterophilous player as "homo-hetero (or hetero-homo)".

First of all, let us consider case 3 as a case of reference, where homophilous players and heterophilous players equally exist. In Fig. 1-6, 14, and 15, case 3 is expressed by green line.

If we look at Fig. 5, 6, and 15, we can see that the number 

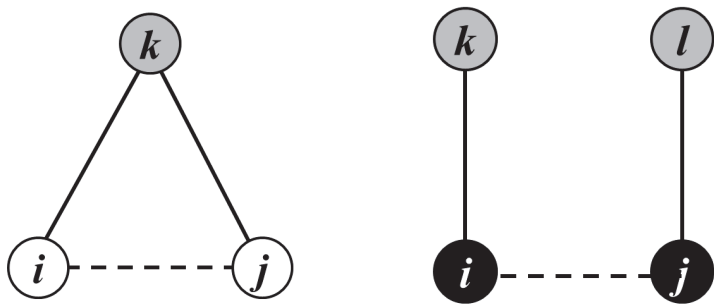

Fig. 7. The condition where ho- Fig. 8. The condition where hetmophilous players $i$ and $j$ add a link erophilous players $i$ and $j$ add a link $i j$ ij

of hetero-hetero links (Fig. 6) is highly increased in an early stage. Then, following it, the number of homo-homo links (Fig. 14) gets increased. After homo-homo links, the number of homo-hetero links (Fig. 5) grows. Thus, it is the basic pattern that the links get connected in order of 1) hetero-hetero, 2) homo-homo, 3) homo-hetero. The reason of this pattern is shown below.

Now, in the case where players $i$ and $j$ are not connected $(i j \notin g)$, forming link $i j$ by both players' rational choices requires one of the conditions depicted in the Fig. 7 and 8. In the figures, white nodes express homophilous players, while black nodes express heterophilous players. Dark nodes can be either of homophilous and heterophilous players. When players $i$ and $j$ are homophilous (Fig. 7), in order to be connected, they must already have common neighbor $k$, which means that $S_{i j}=S_{j i} \geq 1$ should be satisfied for forming the link $i j$. Meanwhile, When players $i$ and $j$ are heterophilous (Fig. 8), in order to be connected it is required that for player $i$ adding a link with $j$ should bring new knowledge from $l$ and also that for the player $j$ forming the link with $j$ should bring new knowledge from $k$. This means that both $D_{i j} \geq 1$ and $D_{j i} \geq 1$ should be satisfied for forming the link $i j$.

To reach these conditions from the empty network, at least two error/mutation are required for making links such as link $i k$ and $j k$ in Fig. 7 and link $i k$ and $j l$ in Fig. 8. In terms of probability, the latter network $g=\{i k, j l\}$ is more likely to be created, since $l(\neq k)$ is allowed to be any player. Thus, in an early stage of dynamic process hetero-hetero links are more likely to be connected, and subsequently homo-homo links are formed.

Next, let us look at link formation between players with distinct preferences. The case where heterophilous player $i$ and homophilous player $j$ make link $i j$ by rational choice for the first time should simultaneously satisfy the following two conditions.

Condition 1

For a heterophilous player $i$, player $j$ already maintains a link with $k$ who is not $i$ 's neighbor.

Condition 2

For a homophilous player $j$, player $i$ already maintains a link with $l$ who is also $j$ 's neighbor.

The situation where both condition 1 and 2 are simulta- neously satisfied depends on whether each player $k$ and $l$ is homophilous or heterophilous. Let us call each situation "situation A", "situation B", "situation C" and "situation D", respectively, as depicted in Fig. 9-12. In Fig. 9-12 white nodes are homophilous players and black nodes are heterophilous players.

Here, let us assume the case where a homo-hetero link is formed for the first time by rational decision making. This assumption requires that homo-hetero links in situations AD have already been made by error/mutation. Situations C and $\mathrm{D}$ has two homo-hetero links respectively, so whether situations $\mathrm{C}$ and $\mathrm{D}$ exist heavily depends on the probability of error/mutation. In other words, the situation where homohetero links are likely to be added by error/mutation is the situation where homo-hetero links grow faster. Additionally, with regard to homo-homo links, situation A has two homohomo links, each situation B-D has one link, so maintaining homo-homo links is also much influential in forming link $i j$.

Hence, case 3 has the following structure where homo-homo links, hetero-hetero links and homo-hetero links are connected.

- In an early stage

Hetero-hetero links grow faster from the empty network because the situation as depicted in Fig. 8 is likely to appear. Subsequently, homo-homo links get formed.

- In a later stage

Since homo-homo links already exist, situations A-D are likely to appear, which leads homo-hetero links to be connected.

On the basis of case 3 , let us consider cases 1 and 2 where the proportion of homophilous players is small. In Fig. 1-6, 14, and 15 , case 1 is expressed by red line and case 2 by orange line.

- In an early stage

The proportion of heterophilous players is so large that heterophilous players are more likely to have links formed by error/mutation. Thus, the situations depicted in Fig. 8 are more likely to exist, so hetero-hetero links highly increase in an early stage. This leads a heterophilous player's degree to reach a higher level. For this reason, the red and orange lines remain at a higher level until around $t=2000$ in Fig. 2. As shown in Fig. 7, homo-homo links are essentially difficult to be formed, and moreover the proportion of homophilous players is so small that homophilous players are less likely to have links formed by error/mutation. For this reason, homo-homo links have not been added so much across time.

- In a later stage

The number of homo-homo links, which affect so much homo-hetero link formation, is small, but the number of hetero-hetero links is so large that situations B and D is more likely to appear. Thus, homo-hetero links grow gradually.

Finally, let us consider cases 4 and 5 where the proportion of homophilous players is large. In Fig. 1-6, 14, and 15, case 4 is expressed by blue line and case 5 is expressed by black line. 


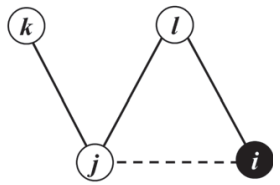

Fig. 9. Situation A

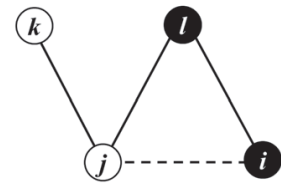

Fig. 10. Situation B

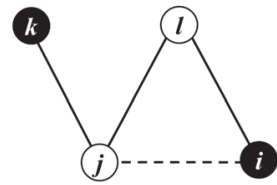

Fig. 11. Situation C

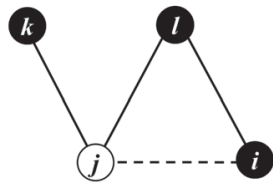

Fig. 12. Situation D

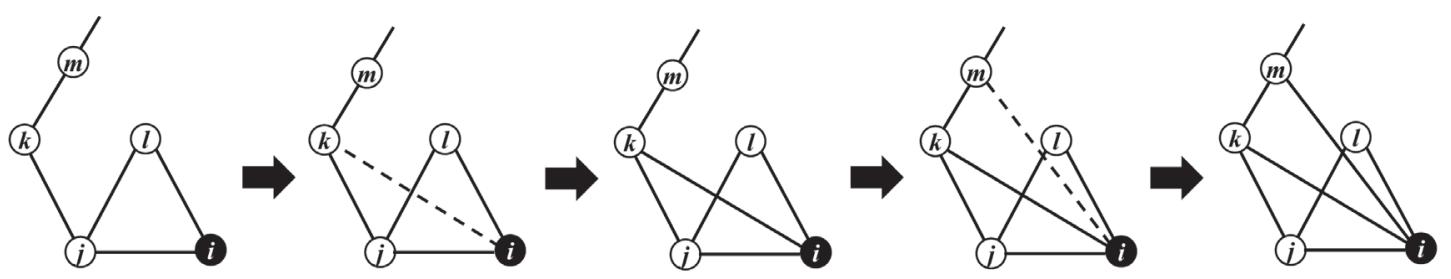

Fig. 13. The process of the increase in one heterophilous player's degree

- In an early stage

The proportion of homophilous players is so large that homophilous players are more likely to have links formed by error/mutation. Thus, the situation depicted in Fig. 7 is more likely to exist, so homo-homo links highly increase in an early stage. On the other hand, the proportion of heterophilous players is so small that the situation depicted in Fig. 8 is less likely to appear. For this reason, hetero-hetero links have not been added so much across time.

- In a later stage

The number of homo-homo is large, so situation $\mathrm{A}$ is the most likely to exist and subsequently situations B and C are more likely to exist. Thus, homo-hetero links grow largely. As homo-hetero links grow, because of the small proportion of heterophilous players, the number of heterohomo links that one heterophilous player has increases. Furthermore, let us look at the case after hetero-homo link $i j$ is added in situation A (the leftmost in Fig. 13). This stage already has a lot of homo-homo links, so it is likely that homophilous player $k$ and homophilous player $m$ are connected. Thus, we see the same situation with A (the second from the left in Fig. 13), so players $i$ and $k$ add link $i k$ by their rational choice (the third from the left in Fig. 13). Then, player $m$ is likely to have other homo-homo links, and again we see the same situation with A (the fourth from the left in Fig. 13), which can lead players $i$ and $m$ to be connected (the fifth from the left in Fig. 13); repeating this process gets heterophilous player $i$ 's degree increased. For this reason, the blue and black lines appear at a higher level since around $t=2000$ in Fig. 2.

\section{Role of Festivals}

\section{A. Network Formation with Festivals}

This section considers a community with festivals. Festivals seem to exist in a context independent of daily jobs or hobbies of residents, so the way of adding links through festivals seems to be different from that dependent on utility function already formulated.

Thus, after the 1st - 4th steps of dynamic process shown in the previous section we add the following 5th step, in which festivals allow players to be connected at random without mutual consent.

5 th

If the link $i j$ is not in the network, then the link $i j$ is added at random without mutual consent. In other words, if the link $i j$ is not in the network $g_{1}^{t}$, then it is added with probability $\eta(0<\eta<1)$ while it is not added with probability $1-\eta$. Additionally, when the link $i j$ is added, if there are no links except $i j$, remove link $i j$, otherwise, remove another link $k l$ randomly identified. The above process generates network $g_{2}^{t}$.

To be more specific, the network $g_{2}^{t}$ is obtained as follow:

- If $i j \notin g_{1}^{t}$, then $g_{2}^{t}=g_{1}^{t}+i j-k l$ (, where if $g_{1}^{t}=$ $\emptyset, k l=i j$, otherwise $k l \neq i j$ ) with probability $\eta$, and $g_{2}^{t}=g_{1}^{t}$ with $(1-\eta)$. Otherwise, $g_{2}^{t}=g_{1}^{t}$.

The network $g_{2}^{t}$ that is determined through the above process is the network at the end of period $t$. This network is also the network at the beginning of period $t+1$. After period $t+1$, the above process is repeated.

Note that the reason why we delete link $k l \in g_{1}^{t}$ as well as add link $i j$ is as follows: Adding one link in the 5th step without deletion obviously increases the number of links in networks. We need to avoid this obvious result because our aim is to investigate the true long run effect of adding a link in the 5th step on network formation. So we remove link $k l$.

In numerical simulation, we specify the probability of adding a link through festivals as $\eta=0.05$, and use same functions and parameters in the previous section. 

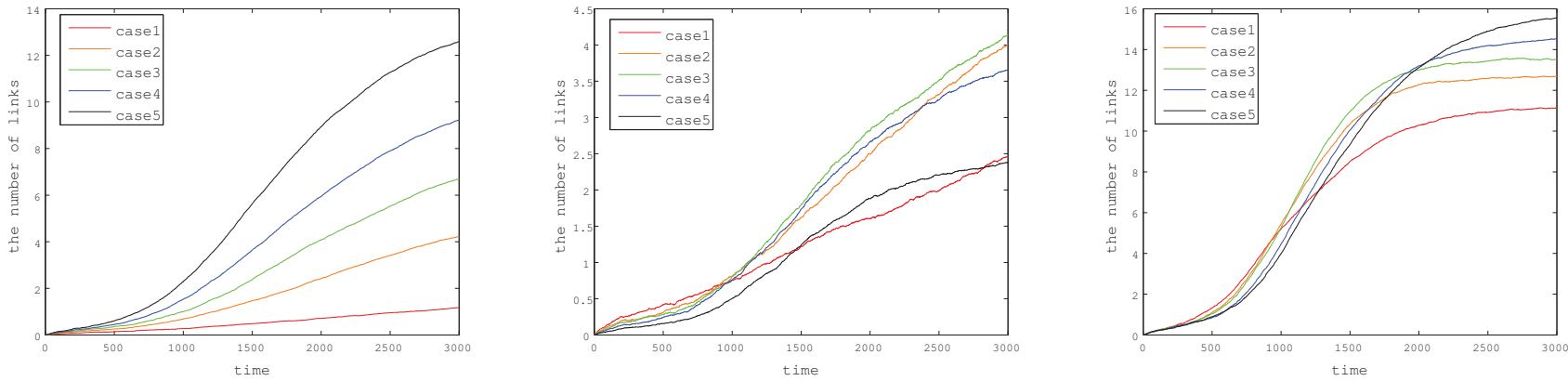

Fig. 14. The number of links that one homophilous Fig. 15. The number of links that one homophilous Fig. $16 . \quad$ One homophilous player's degree in a player has with homophilous players player has with heterophilous players community with festivals
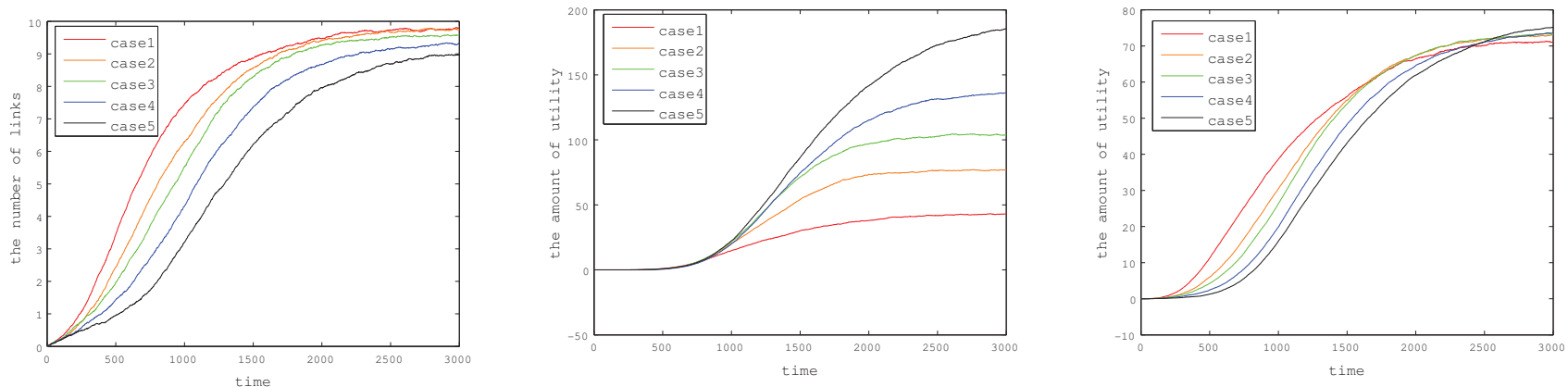

Fig. 17. One heterophilous player's degree in a Fig. 18. One homophilous player's net utility Fig. $19 . \quad$ One heterophilous player's net utility

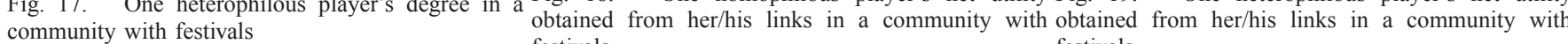
festivals festivals

\section{B. Results}

Given that festivals exist, the 5th step forms a link independently of a pair's utility of day-to-day life and then deletes another link. Here, the series of the process does not change the number of links in a network, so we cannot see the growth of a network within the period. However, comparing Fig. 1-4 with Fig. 16-19 respectively, we can observe the increase in degree of each homophilous player and heterophilous player in every case when festivals exist, which means networks grow in the long run with the presence of festivals. We can also observe the long-term increase in each homophilous player's and heterophilous player's net utility. This tendency is remarkable especially in cases 1 and 2, where the proportion of homophilous players is small.

Let us consider the reason of the above phenomena. In the case without festivals, link $i k$ and $j k$ in Fig. 7, link $i k$ and $j l$ in Fig. 8, and homo-hetero links in Fig. 9-12 are never added with mutual consent under utility function in day-to-day life but are added only by error/mutation. However, in the case with festivals, festivals create a link regardless of utility function in day-to-day life, and consequently, link $i j$ in Fig. 7-12 is more likely to be added with mutual consent under utility function in day-to-day life.

Once these links are formed, other links become connected with mutual consent, as is shown before in Fig. 13. Mean- while, new links always generate the positive externality on homophilous players, so more and more homo-homo links are also added with mutual consent. When festivals do not exist, networks in cases 1 and 2 have fewer links, so the above effect of link addition would be much greater.

In summary, in terms of network formation, it is the value of festivals that forms high potential links which produce a negative effect on utility of day-to-day life in the short term but accelerate autonomous network formation and generate a large amount of utility in the long term.

\section{CONClusion}

This study formulated a stochastic dynamics of link formation based on the notion of improving paths in a community, assuming that 1) both "homogeneity" and "heterogeneity" of others' knowledge and experience are endogenously determined, and 2) the players have a variety of preferences. We considered that the role of festivals is to allow players to add links randomly regardless of their utility of day-to-day life, and then we investigated the change of network dynamics with and without festivals by means of numerical simulation.

When no festivals exist, we found that links between players with different preferences were the least likely to appear. However, when festivals exist, we found that even if festivals add links with negative utility in the short run between players with different preferences, these links can 
be triggers that accelerate autonomous network formation not only between heterophilous and homophilous players but also between homophilous players. These links can also produce a large amount of utility in the long run. In terms of network formation, we can emphasize that to add these kinds of links is the value of festivals.

Interesting topics for future works are as follows. Firstly, the model should be extended to take into account endogenously determined preferences of players, while our current model assumes the exogenous preference. Residents' preferences seem to depend largely on their position in networks or experience of festivals. Secondly, the model, which currently formulates festivals in the form of one-to-one meetings, should also include simultaneous link formation because festivals are implemented by the collective actions of many people.

\section{ACKNOWLEDGMENT}

The authors would like to thank Dr. Subhajyoti Samaddar for helpful comments and suggestions.

\section{REFERENCES}

[1] M. S. Y. Chwe, Rational Ritual: Culture, Coordination, and Common Knowledge, Princeton University Press, 2001.

[2] D. Easley and J. Kleinberg, Networks, Crowds, and Markets: Reasoning about a Highly Connected World, Cambridge University Press, 2010.

[3] A. Calvo-Armengol and M. O. Jackson, "The effects of social networks on employment and inequality," American Economic Review, pp. 426-454, 2004.

[4] C. Ballester, A. Calvo-Armengol, and Y. Zenou, "Who's who in networks. wanted: the key player," Econometrica, Vol. 74, No. 5, pp. 1403-1417, 2006.

[5] E. Durkheim, Les formes elementaires de la vie religieuse, Presses universitaires de France, 1937.

[6] V. W. Turner, Dramas, Fields, and Metaphors: Symbolic Action in Human Society, Cornell University Press, 1975.

[7] K. Shimane, "Cultural apparatus as determiners for exceptional times: Beyond the Sociology of Everyday Life," (in Japanese) in Cultural Apparatus as Determiners for Exceptional Times, K. Shimane and M. Fujimura, Eds., Hokuju Shuppan, pp. 16-37, 2001.

[8] C. Johnson and R. P. Gilles, "Spatial social networks," Review of Economic Design, Vol. 5, No. 3, pp. 273-299, 2000.

[9] E. Droste, R. P. Gilles, and C. Johnson, "Evolution of conventions in endogenous social networks," CentER, Tilburg University, The Netherlands, 2000, unpublished.

[10] R. Iijima and Y. Kamada, "Social distance and network structures," Harvard University, 2010, unpublished.

[11] P. F. Lazarsfeld and R. K. Merton, "Friendship as a social process: A substantive and methodological analysis", Freedom and Control in Modern Society, pp. 18-66, 1954.

[12] M. McPherson, L. Smith-Lovin, and J. M. Cook, "Birds of a feather: Homophily in social networks", Annual Review of Sociology, pp. 415444, 2001.

[13] M. O. Jackson and A. Watts, "The evolution of social and economic networks," Journal of Economic Theory, Vol. 106, No. 2, pp. 265-295, 2002.

[14] S. Currarini, M. O. Jackson, and P. Pin, "An economic model of friendship: Homophily, minorities, and segregation," Econometrica, Vol. 77, No. 4, pp. 1003-1045, 2009.

[15] F. Fu, M. A. Nowak, N. A. Christakis, and J. H. Fowler, "The evolution of homophily," Scientific reports, Vol. 2, 2012.

[16] M. O. Jackson and A. Watts, "On the formation of interaction networks in social coordination games," Games and Economic Behavior, Vol. 41, No. 2, pp. 265-291, 2002. 